Geopolítica(s) Revista de estudios sobre espacio y poder ISSN: 2172-3958

http://dx.doi.org/10.5209/GEOP.54217

\title{
David Slater: un geógrafo político comprometido
}

\section{Heriberto Cairo}

Cómo citar: Cairo, Heriberto (2016) "David Slater: un geógrafo político comprometido". Geopolítica(s). Revista de estudios sobre espacio y poder, vol. 7, núm. 2, 175-180.

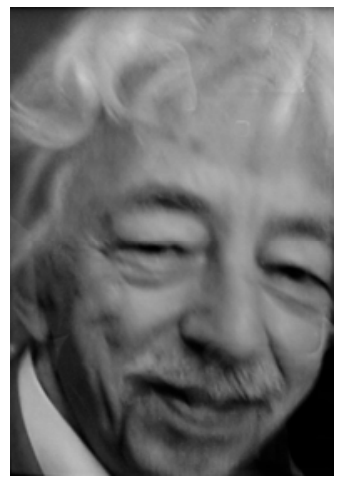

Tras las pérdidas de Edward Soja y Doreen Massey, que reseñábamos en el anterior número de Geopolítica(s), nos acaba de sacudir la muerte, el 20 de octubre pasado, de David Slater, miembro de nuestro Consejo Asesor, uno de los más destacados geógrafos políticos de las últimas décadas $\mathrm{y}$, sobre todo o por debajo de todo, maestro y amigo.

Enseñó en universidades de Brasil, Canadá, Chile, España, Estados Unidos, México, Países Bajos, Perú, Reino Unido, Sri Lanka y Tanzania. Sus puestos más fijos y duraderos fueron en el Centro de Estudios y Documentación Latinoamericanos (CEDLA) de la Universidad de Amsterdam y en el departamento de Geografía de la Universidad de Loughborough (1994 hasta ahora). Son muchos lugares donde impartió su magisterio, muchos de ellos fuera de la región del centro académico, siempre con el deseo de colaborar en los proyectos que le presentábamos y dispuesto a "perder" su tiempo con nosotros y nosotras, sin esperar recompensas importantes. Esa forma de hacer Academia, que está por encima de los intereses más personales - sean estos de la índole que sean-, ha hecho avanzar en muchos sitios una geografía política comprometida con la democracia y la resistencia a la injusticia y a la dominación. 
Entre 1999 y 2004 fue coeditor de Political Geography, la revista fundada por Peter J. Taylor que se ha convertido en la más importante de este campo de conocimiento. En el primer número del volumen 19, Slater (2000) escribía un editorial en el que se expresaban con claridad algunas de sus posiciones ante la vida, la Academia y, por supuesto, la geografía política en tanto que disciplina. Percibía los tremendos cambios que se habían producido en los últimos años, y los entendía como una oportunidad:

En un mundo de creciente desigualdad y turbulencia, de conflictos sociales y movimientos de resistencia, de politización del cambio medioambiental, de repensar la gobernanza y la democracia, de resurgimiento de las preocupaciones ético-políticas en relación a los derechos humanos, el lugar de los pueblos indígenas y el significado global de los temas de género y "raza", está claro que hay un abundante suministro de materia prima y recursos para nuevas geografías políticas (Slater, 2000: 3).

Tres preocupaciones presidían el editorial: 1) mantener e incluso incrementar la pluralidad dentro de la revista; 2) repensar las relaciones sociedad-Estado desde esa plataforma privilegiada, reflexionando sobre la democratización y los movimientos sociales, y 3) superar el etnocentrismo de la geografía política euro-americana abriéndose a la producción de los autores del Tercer Mundo. Hoy en día tienen todavía vigencia, la geografía política sigue siendo en gran medida anglosajona y es difícil abrir reflexiones alternativas, y la pluralidad es difícil de combinar con el compromiso, como pudo comprobar David a los cinco años de coeditar la revista.

La obra de David Slater ha sido amplia y relevante, en particular para el estudio de la geografía política de América Latina, en particular, y de las relaciones NorteSur, en general. Sin ánimo de ser esquemático, creo que se puede estructurar en tres ámbitos de reflexión: el problema del desarrollo/subdesarrollo y la acción del Estado, las relaciones Norte-Sur y el intervencionismo imperialista, y, de forma general, cómo desarrollar una teoría crítica que favoreciese las transformaciones sociales en pos de un mundo más humano.

Uno de sus primeros trabajos "Geography and Underdevelopment" aborda la cuestión del subdesarrollo desarrollando una crítica contundente de las teorías modernizadoras del desarrollo, que mantendrá hasta sus últimos días. En Territory and State Power in Latin America: The Peruvian Case, Slater (1989) aplica al caso peruano sus análisis sobre el desarrollo y en particular su relación con la estructura territorial del Estado. A partir de algunas categorías marxistas ${ }^{1}$ aborda el estudio de la cuestión regional en Perú, el proceso de urbanización y la centralización territorial del Estado. Estados como Perú son para él una variante del Estado capitalista: son "Estados capitalistas periféricos" (Slater, 1989: 231), que intervienen a través de la creación de ideologías territoriales, de la diferenciación regional, de la concentración espacial de recursos del Estado en áreas que son base del capital internacional y nacional, y del establecimiento de procesos de regionalización para el desarrollo. Pero sus capacidades son limitadas, porque están encajados en la estruc-

Interesantemente, la relación entre espacio y Estado la desarrolla a partir de los trabajos de Poulantzas (1973, 1978), que quizás no hayan sido valorados suficientemente en geografía política. 
tura centro-periferia bajo dominio imperial, que sólo se podría superar mediante procedimientos radicales; en sus palabras:

Las experiencias de Cuba y Nicaragua, a pesar de sus incisivos contrastes, demuestran, sin ningún género de duda, que una ruptura revolucionaria de la dominación internalizada del capital internacional, y específicamente del de los EE UU, proporciona una condición necesaria si no suficiente para una efectiva reorganización territorial de la economía y la sociedad, realizada en función de afrontar las necesidades humanas (Slater, 1989: 235).

La preocupación por el estudio de los movimientos sociales, de nuevo de forma particular en América Latina, también constituye otro de los rasgos característicos de su obra. De ahí surgió New Social Movements and the State in Latin America (Slater, 1985a), a partir de un taller celebrado en Amsterdam, en el CEDLA, con el objetivo de traer el debate que se estaba produciendo en América Latina a Europa, y donde fueron invitados varios expertos latinoamericanos y europeos (E. Laclau, J. L. Coraggio, T. Evers, E. Henry, M. Molyneux, entre otros). Se trataba de entender qué cambios introducían en la política los "nuevos" movimientos sociales, que Slater (1985b: 3-7) mostraba que eran diferentes a los europeos, aunque hubiesen surgido gracias a la aparición de éstos. En su intervención en el taller, Slater (1985b) ponía en cuestión las tesis de Miliband (1985) que, entre otros, afirmaban que sólo la clase obrera tiene capacidad revolucionaria, y apoyándose en Laclau y Mouffe (1985) sostenía la capacidad transformadora de los movimientos latinoamericanos que luchaban por una democracia radical. Muestra aquí Slater el alejamiento de alguno de los anquilosados clichés de la izquierda marxista, e incluso de algún neomarxista, y su apuesta por explorar nuevos caminos, sin debilitar su compromiso con las teorías críticas para el cambio social.

Geopolitics and the Post-colonial: Rethinking North-South Relations (Slater, 2004) es a mi juicio su obra más completa y funciona como un parteaguas articulador de sus preocupaciones pasadas y futuras. Retoma sus temas tradicionales, muestra su actualidad y abre las vías para la futura reflexión. Es lo que hace, por ejemplo, con la teoría de la dependencia: no sólo muestra su interés para explicar lo que las teorías de la modernización no pueden explicar, sino que abre nuevas vías de indagación con ella, más allá de lo económico, pero sin desecharlo. En sus propias palabras:

Este libro ha nacido de un interés de larga duración en las relaciones Norte-Sur y en su conexión crucial con la potencia de las intervenciones geopolíticas. El énfasis analítico reside en ideas y temas que han sido de importancia clave para esta región y que todavía lo siguen siendo. El enfoque que he tomado está basado en buena medida en el pensamiento post-estructuralista y post-colonial, con vínculos a la teoría del desarrollo crítica, y la contextualización se hace principalmente a través de ejemplos ilustrativos seleccionados de la trayectoria de las relaciones entre EE UU y América Latina (Slater, 2004: vii).

El surgimiento del neoliberalismo es descrito con maestría y, para cerrar, revisa, de forma comprometida pero rigurosa, lo que denomina "archipiélagos de pensa- 
miento crítico" (Slater, 2004: 115 y ss.): la teoría dependentista, el pensamiento descolonial, la "utopía" zapatista... De nuevo los movimientos sociales abren la posibilidad de transformación de un sistema que ancla sus bases en la diferencia colonial e imperial.

Finalmente, si hubiera que identificar el principal objeto de sus investigaciones más recientes, creo que habría que señalar la "imperialidad" (imperiality). Sus reflexiones (Slater, 2009, 2010a, 2010b) sobre esta cuestión son claves para entender las formas de dominio en el sistema mundial actual. En primer lugar porque muestra que el fin del imperialismo formal asociado a la colonización no supone el fin de un determinado tipo de relación geopolítica entre los países imperiales centrales y los periféricos. En este sentido,

mientras que el imperialismo puede ser pensado en términos de estrategia, práctica y justificación del poder invasivo de un Estado occidental sobre otros Estados predominantemente no occidentales, cuya soberanía política es así minada, la imperialidad se refiere al sentimiento, privilegio y derecho percibido de ser imperial o de defender un modo de vida imperial en el que la "invasividad" geopolítica se legitima (Slater, 2010b: 195).

Las oposiciones Norte-Sur o centro-periferia son las expresiones de la "diferencia que se ancla en el poder imperial - que la diferencia imperial es la diferencia entre sociedades imperiales y sociedades imperializadas, $y$, a pesar de las heterogeneidades complejas que existen entre ambas categorías, hay una profunda relación asimétrica que hunde sus raíces en una historia de saber y poder" (Slater, 2010a: 201-202). Por lo tanto, "los parámetros de la razón imperial están todavía activos y la orientación dirigente de una mentalidad imperial no ha sido superada" (Slater, 2010b: 202).

Slater construye el concepto de "imperialidad" de forma relativamente paralela a cómo Aníbal Quijano — sociólogo peruano- conforma el de "colonialidad"2. Ambas son un aporte fundamental al estudio de los países periféricos desde las ciencias sociales, permitiendo entender las continuidades y los cambios que se producen en ellos desde la irrupción del poder imperial, que destruye las estructuras y prácticas sociales previas y las sustituye por otras, que perviven a la independencia formal.

David Slater (2008) intenta también abrir un debate sobre importantes aspectos de la intersección entre poder, conocimiento y geopolítica en el contexto del resurgimiento de las visiones imperiales, que hacen urgente la des-colonización de la imaginación geopolítica mediante multidimensionales respuestas y resistencias. Considera la importancia de lo "crítico" en el conocimiento crítico, y desnuda la prevalencia del pensamiento euro-estadounidense. La des-colonización del cono-

Según la definición de Aníbal Quijano (2000a; 2000b) la “colonialidad del poder”, que está basada en la clasificación de las razas, permite entender cómo en los Estados latinoamericanos independientes se mantuvo (y mantiene) la sociedad colonial (con la salvedad de Haití): "La «racialización» de las relaciones de poder entre las nuevas identidades sociales y geo-culturales, fue el sustento y la referencia legitimatoria fundamental del carácter eurocentrado del patrón de poder, material e intersubjetivo. Es decir, de su colonialidad" (Quijano, 2000b: 374). 
cimiento tiene que incluir entonces, a su juicio, una crítica continua de la ambición y la arrogancia imperial.

Continuará con esta línea de reflexión hasta su final. Siempre activo, en el mes de julio pasado estuvo con nosotros en Madrid en el curso de verano de la Universidad Complutense sobre "La crisis y la reconfiguración de la geopolítica mundial", su conferencia se tituló "Challenging Imperial Reason: Re-framing the Way We Think about Global (Geo)politics". Allí nos anunció que formaba parte de un proyecto mayor, quizás un libro, sobre Geopolitics and the Dynamic of Imperial Power.

Cuando le conocí, en el congreso de la International Political Science Association en Berlín en 1994, se quejaba ante quien quisiera oírle de las tasas de inscripción, que consideraba inusualmente altas, especialmente para los académicos que no provenían de países del centro. De forma amable, pero contundente, denunciaba las dificultades que los académicos y estudiantes de los países periféricos tenían para participar en estos eventos y lo poco sensibles que eran los dirigentes de estas asociaciones a estos problemas. Nos ha dejado una persona noble de espíritu, pero siempre nos quedará su recuerdo y su maestría.

\section{Bibliografía}

Laclau, Ernesto, and Mouffe, Chantal (1985) Hegemony and Socialist Strategy - Towards a Radical Democratic Politics. Londres: Verso.

Miliband, Ralph (1985) “The New Revisionism in Britain”. New Left Review, núm. 150, 526.

Poulantzas, Nicos (1973) Political Power and Social Classes. Londres: New Left Books.

Poulantzas, Nicos (1978) State, Power, Socialism. Londres: New Left Books.

Quijano, Aníbal (2000a) "Colonialidad del poder, eurocentrismo y América Latina”, en E. Lander (comp.): La colonialidad del saber: eurocentrismo y ciencias sociales. Perspectivas latinoamericanas. Buenos Aires: CLACSO, 201-246.

Quijano, Aníbal (2000b) "Colonialidad del poder y clasificación social". Journal of WorldSystems Research, vol. VI, núm. 2, 342-386.

Slater, David (1973) "Geography and Underdevelopment". Antipode: A Radical Journal of Geography, vol. 5, núm. 3, 21-32.

Slater, David (1982) Capitalismo y desarrollo regional: Cinco ensayos críticos sobre la organización del espacio en el tercer mundo. Amsterdam: CEDLA.

Slater, David (ed.) (1985a) New Social Movements and the State in Latin America. Amsterdam: CEDLA.

Slater, David (1985b) "Social Movements and a Recasting of the Political", en D. Slater (ed.) New Social Movements and the State in Latin America. Amsterdam: CEDLA, 125.

Slater, David (1989) Territory and State Power in Latin America: The Peruvian Case. Nueva York: Palgrave Macmillan.

Slater, David (2000) "Editorial statement. The process and prospect of political geography". Political Geography, vol. 19, núm. 1, 1-3.

Slater, David (2004) Geopolitics and the Post-colonial: Rethinking North-South Relations. Malden (MA) y Oxford: Blackwell. 
Slater, David (2008) "Repensando la geopolítica del conocimiento: desafiando las visiones imperiales", en H. Cairo y W. Mignolo (eds.) Las vertientes americanas del pensamiento y el proyecto des-colonial. Madrid: Trama editorial / GECAL, 153-174.

Slater, David (2009) "Exporting Imperial Democracy: Critical Reflections on the US Case". Human Geography, vol. 2, núm. 3, 24-36.

Slater, David (2010a) "Rethinking the Imperial Difference: towards an understanding of US-Latin American encounters". Third World Quarterly, vol. 31, núm. 2, 185-206.

Slater, David (2010b) "The imperial present and the geopolitics of power". Geopolítica(s). Revista de estudios sobre espacio y poder, vol. 1, núm. 2, 191-205.

Slater, David, \& Taylor, Peter J. (eds.) (1999) The American Century: Consensus and Coercion in the Projection of American Power. Oxford: Wiley-Blackwell. 